\title{
COMPLETE SETS OF DIAGONAL OPERATORS IN QUANTUM-STATISTICAL MECHANICS
}

\author{
W. J. CASPERS and H. P. VAN DE BRAAK \\ Technische Hogeschool Twente, Enschede, Nederland \\ P. W. VERBEEK and J. C. VERSTELLE \\ Kamerlingh Onnes Laboratorium, Leiden, Nederland
}

Received 8 january 1971

\section{Synopsis}

Complete sets of diagonal operators, i.e. operators commuting with the hamiltonian of a physical system, are constructed. In terms of these sets all diagonal operators can be written as a series, the uniform convergence of which is studied for infinitely large systems. This uniform convergence is introduced as a possible criterion for ergodicity.

1. Introduction. A central problem in the quantum theory of irreversible processes is to find the so-called secular part of the self-adjoint operator corresponding with a variable of a physical system, the motion of which is described by a hamiltonian $H$. It is easy to show that the set of all secular, self-adjoint operators, i.e. operators commuting with $H$, constitute a vector space, being a subspace of the space of all sclf-adjoint opcrators. The sccular part of any hermitean operator is simply the projection on this subspace.

We restrict ourselves mainly to the case that the eigenvalues of $H$ are nondegenerate; for this case secular operators may be called diagonal. Degeneracy may occur as level crossing for a hamiltonian that is a linear function of a real parameter.

For a finite-dimensional Hilbert space it is easy to find a basis for the subspace of diagonal operators. This basis is given by the first $n$ powers of $H, n$ being the dimension of the Hilbert space. Apart from a gencral method for the construction of an orthogonalized basis, this paper is concerned with the convergence or divergence of an expansion of the diagonal part of an operator in terms of this basis, in the limit $N \rightarrow \infty, N$ being the total number of particles in the system. Uniform convergence of this expansion for $N \geq N_{\mathbf{0}}$, $N_{0}$ being a fixed integer $>0$ is a sufficient condition for the ergodicity of the corresponding physical quantity ${ }^{1,2}$ ).

A well-known example of a divergent series expansion is given by systems for which the so-called adiabatic and isolated susceptibilities show a differ- 
ence of the order of the number of particles. In the literature these susceptibilities usually refer to the linearized quasistatic response of a magnetic system for a slowly varying external magnetic field ${ }^{3-5}$ ).

In section 2 we define a complete set of diagonal, orthogonalized operators for a given hamiltonian $H$ and we introduce a positive definite metric, which makes it easy to find a series expansion for the diagonal part of any selfadjoint operator. We restrict ourselves to finite-dimensional Hilbert spaces. The possible convergence of this series expansion for $N \rightarrow \infty$ is discussed in an introductory way in section 3 , together with the relation between this mathematical property and the ergodicity of the corresponding physical quantity.

In section 4 we give a discussion of the relation between non-ergodicity of a quantity $B$ and level crossing for a large system with a hamiltonian $H=A+h B$ that is a linear function of a real parameter $h$. Noncrossing has been made a fundamental assumption in an attempt to prove the identity of the adiabatic and isolated susceptibilities 5,6 ). In a recent paper by one of the authors a relation is discussed between the number of level crossings and the degree of possible basis vectors of the space of diagonal operators, i.e. the degree in the parameter $h^{2}$. Nonergodicity in the literature always corresponds with a basis that is not given by the first $n$ powers of $H$.

Finally in section 5 ergodicity is discussed as a consequence of the uniform convergence of the series introduced in section 2 , for $N \geq N_{0}>0$.

2. A complete set of diagonal operators. In ref. 7 it was shown that for an $n$-dimensional Hilbert space a complete set of diagonal operators is given by $I, H, H^{2}, \ldots, H^{n-1}$, in which $I$ is the identity operator. The condition of nondegeneracy is implicit, unless otherwise stated, also in the present work. For every diagonal (self-adjoint) operator $F$ the operator equation

$$
F=\sum_{k=0}^{n-1} a_{k} H^{k}
$$

can always be solved for the real numbers $a_{k}$. This equation corresponds with $n$ linear equations $F_{i}=\sum_{k=0}^{n-1} a_{k} \varepsilon_{i}^{k}, i=1,2, \ldots n$, the index $i$ numbering the $n$ different eigenvalues of $H, \varepsilon_{1}, \varepsilon_{2}, \ldots, \varepsilon_{n}$. The $F_{i}$ are the corresponding (diagonal) clements of $F$.

The set of $n$ equations for the $a_{k}$ has a unique solution, the determinant of the set being different from zero, because it equals the well-known Vandermonde determinant for the $n$ different quantities $\varepsilon_{i}$. As a consequence of the fact that the sum of two diagonal operators is also diagonal and that multiplication of a real diagonal operator by a real number again gives an operator of the same set, we may conclude that this set of all real diagonal operators constitutes a vector space.

Our first problem is to construct an orthogonal basis from the original one 
$I, H, \ldots, H^{n-1}$. In order to do this we have to define an inner product of two vectors in the operator space in the most convenient way. In problems of irreversible statistical mechanics or ergodic theory we are most times interested in the Boltzmann average of the square of diagonal operators and so it is reasonable to include the Boltzmann factor in the definition of the inner product ${ }^{1}$ ). For hermitean operators this results in a pusitive definite metric and the square of a vector being zero implies that this vector is the zero element. So the inner product obeys

$$
\begin{aligned}
\langle F G\rangle & =\frac{\operatorname{Tr} \mathrm{e}^{-\beta H} F G}{\operatorname{Tr} \mathrm{e}^{-\beta H}}=\langle G F\rangle, \quad\left\langle F^{2}\right\rangle=\frac{\operatorname{Tr} \mathrm{e}^{-\beta H} F^{2}}{\operatorname{Tr} \mathrm{e}^{-\beta H}} \geq 0, \\
\beta & =1 / k T .
\end{aligned}
$$

Now an orthogonal basis $e_{i}, i=1,2, \ldots, n$, is easily constructed and most conveniently represented in a determinantal notation

$$
\begin{aligned}
& e_{1}=I, \quad e_{2}=\left|\begin{array}{cc}
I & H \\
& \\
\langle I\rangle & \langle H\rangle
\end{array}\right|, \quad e_{3}=\left|\begin{array}{ccc}
I & H & H^{2} \\
\langle I\rangle & \langle H\rangle & \left\langle H^{2}\right\rangle \\
\langle H\rangle & \left\langle H^{2}\right\rangle & \left\langle H^{3}\right\rangle
\end{array}\right|, \ldots \\
& e_{n}=\left|\begin{array}{ccccc}
I & H & H^{2} & \ldots & H^{n-1} \\
\langle I\rangle & \langle H\rangle & \left\langle H^{2}\right\rangle & \ldots & \left\langle H^{n-1}\right\rangle \\
\langle H\rangle & \left\langle H^{2}\right\rangle & \left\langle H^{3}\right\rangle & \ldots & \left\langle I^{n}\right\rangle \\
\vdots & & & & \\
\left\langle H^{n-2}\right\rangle & \left\langle H^{n-1}\right\rangle & \left\langle H^{n}\right\rangle & \left\langle H^{2 n-3}\right\rangle
\end{array}\right|
\end{aligned}
$$

The orthogonality $\left\langle e_{i} e_{j}\right\rangle=0, i \neq j$, is easily demonstrated: We may take $i<j$ and for this case $e_{i}$ is a linear combination of $I, H, \ldots H^{i-1}$ with all power exponents being smaller than $j-1$, the highest one in $e_{j}$. If we show that all these powers $H^{k}, 0 \leq k \leq i-1$, have an inner product with $e_{j}$ that equals zero we know that $\left\langle e_{i} e_{j}\right\rangle=0$. But it is easily seen that the inner products $\left\langle H^{k} e_{j}\right\rangle$ result in a determinant with two identical rows and so it equals zero indeed. This reasoning completes our proof.

In order to evaluate the diagonal part of a given operator it is also necessary to know the square of the different basis vectors $e_{i}$. We have already shown that $\left\langle H^{k} e_{j}\right\rangle=0$ for $k\langle j-1$, so it immediately follows that

$$
\left\langle e_{i}^{2}\right\rangle=\mathscr{D}_{i-1} \mathscr{D}_{i}, \quad i=1,2, \ldots n,
$$

in which expressions the $\mathscr{D}_{i}$ are given by

$$
\mathscr{D}_{i}=\left|\begin{array}{ccccc}
\langle I\rangle & \langle H\rangle & \left\langle H^{2}\right\rangle & \ldots\left\langle H^{i-1}\right\rangle \\
\langle H\rangle & \left\langle H^{2}\right\rangle & \left\langle H^{3}\right\rangle & \ldots\left\langle H^{i}\right\rangle \\
\left\langle H^{2}\right\rangle & \left\langle H^{3}\right\rangle & \left\langle H^{4}\right\rangle & \ldots\left\langle H^{i+1}\right\rangle \\
\vdots & \vdots & & \\
\left\langle H^{i-1}\right\rangle & \left\langle H^{i}\right\rangle & \left\langle H^{i+1}\right\rangle & \ldots\left\langle H^{2 i-2}\right\rangle
\end{array}\right|, i \geq 1 ; \mathscr{D}_{0}=1 .
$$


Now as a consequence of the completeness of the set $I, H, \ldots H^{n-1}$ the diagonal part of an operator $B$ can be written in the form

$$
B_{\text {diag }}=\sum_{i=1}^{n} \frac{\left\langle B e_{i}\right\rangle}{\left\langle e_{i}^{2}\right\rangle} e_{i},
$$

in which formula the inner product $\left\langle B e_{i}\right\rangle$ of a diagonal operator and one that is in general nondiagonal, is also defined by formula (1). Also in this case we have $\left\langle B e_{i}\right\rangle=\left\langle e_{i} B\right\rangle$. In the statistical problems we mentioned in the introduction, the fluctuation of a quantity $B_{\text {diag }}$ e.g., the fluctuation of the diagonal part of the magnetization of a system, is of great interest and for this fluctuation we may write down a series expansion on the basis of (5)

$$
B_{\text {dlag }}-\langle B\rangle=\sum_{i=2}^{n} \frac{\left\langle B e_{i}\right\rangle}{\left\langle e_{i}^{2}\right\rangle} e_{i},\left\langle\left(B^{\text {diag }}-\langle B\rangle\right)^{2}\right\rangle=\sum_{i=2}^{n} \frac{\left\langle B e_{i}\right\rangle^{2}}{\left\langle e_{i}^{2}\right\rangle} .
$$

In the next section we shall prove that the right-hand side of the second formula is a series expansion in $1 / N, N$ being the total number of particlcs in the system. This second formula is relevant for the problem of the equivalence of the isolated and adiabatic susceptibilities: for $B=M$ the left-hand side corresponds with $k T\left(\chi_{0}-\chi_{\text {is }}\right)$ and the right -hand side with $k T\left(\chi_{0}-\chi_{\mathrm{s}}\right)$, apart from terms of lower order then $\mathcal{O}(N)^{4}$. The symbols $\chi_{0}$, $\chi_{\text {is }}$ and $\chi_{\mathrm{s}}$, respectively, denote the isothermal, isolated and adiabatic susceptibility. As will be stated in more detail in the next section, for nonergodic systems $(1 / N)$ times the series expansion in the second formula of (6) will diverge for $N \rightarrow \infty$; as a consequence of $N \rightarrow \infty$ we have $n(N) \rightarrow \infty$.

3. Cluster expansion for large systems. Now we want to evaluate the determinants $\mathscr{D}_{i}$, given in formula (4), and the inner products

$$
\left\langle B e_{i}\right\rangle=\left|\begin{array}{cccc}
\langle B\rangle & \langle B H\rangle & \ldots & \left\langle B H^{i-1}\right\rangle \\
\langle I\rangle & \langle H\rangle & \ldots & \left\langle H^{i}\right\rangle \\
\vdots & \vdots & \\
\left\langle H^{i-2}\right\rangle & \left\langle H^{i-1}\right\rangle & \left\langle H^{2 i-3}\right\rangle
\end{array}\right|, i=1,2, \ldots n .
$$

We are interested in large systems and in the corresponding asymptotic expressions for $\mathscr{D}_{i}$ and $\left\langle B e_{i}\right\rangle$. For that reason we make a cluster expansion of the quantities $\left\langle H^{i}\right\rangle$ and $\left\langle B H^{i}\right\rangle$, and this is possible for large systems that show an ideal periodicity (periodic boundary conditions) or for which the boundary layers only contain a small fraction of the total number of constituents. We have restricted ourselves to systems with a finite number of states $n$, corresponding with a finite number per constituent i.e. atoms, molecules etc. In the case of magnetic spins $S$ this number equals $2 S+1$.

The total hamiltonian of the system may be written as a sum of terms

$$
H=\sum_{i} H_{i}
$$


corresponding with the unit cells of the lattice, which may contain a number of different constituents. The $H_{i}$ also contains the corresponding interactions in the cell and half of its interactions with the surroundings. Formula (8) expresses the translational symmetry apart from small corrections for the $H_{i}$ of the boundary layers.

For all realistic physical situalions we have $\left\langle H_{i}\right\rangle=\mathcal{O}(1)$ and for the greater part of the system this thermal average does not depend on $i$. So it is possible to introduce the symbol $C_{1}$ for the smallest possible cluster

$$
\langle H\rangle=\sum_{i}\left\langle H_{i}\right\rangle=C_{1}, \quad C_{1}=\mathscr{O}(N) .
$$

The average value $\left\langle H^{2}\right\rangle$ is a double sum and we may distinguish between terms corresponding with neighbouring cells and with cells far apart. So one may understand that $\left\langle H^{2}\right\rangle-\langle H\rangle^{2}$ is also of the order of the number of particles and we write

$$
\left\langle H^{2}\right\rangle=C_{1}^{2}+C_{2}, \quad C_{2}=\mathcal{O}(N) .
$$

A cluster expansion for $\left\langle H^{k}\right\rangle$ may be determined by evaluating the number of ways $k$ factors $H_{i}$ can be arranged in clusters, corresponding with neighbouring cells. Doing so one arrives at the following general expression, making use of the cumulant expansion $\operatorname{method}^{10}$ )

$$
\left\langle H_{k}\right\rangle=k ! \sum_{\sum_{i=1}^{k} n_{j j}=k} \prod_{l} \frac{1}{n_{l} !(l !)^{n_{3}}} C_{l}^{n_{l}}, \quad C_{l}=\mathfrak{O}(N) .
$$

Analogous expressions can be found for the quantities $\left\langle B H^{k}\right\rangle$, but now one has to introduce the additional symbols $D_{m}$ corresponding with a cluster of one factor $B_{i}$ and $m-1$ factors $H_{j}$. One has to bear in mind that also $B=\sum_{i} B_{i}$ is a sum of terms, one for each cell. The cluster expansion for $\left\langle B H^{k}\right\rangle$ takes the form

$$
\begin{aligned}
\left\langle B H^{k}\right\rangle & =k ! \sum_{m=1}^{k+1} \frac{D_{m}}{(m-1) !} \sum_{\substack { k+1-m \\
\begin{subarray}{c}{j-1 \\
n_{j} j=k+1-m{ k + 1 - m \\
\begin{subarray} { c } { j - 1 \\
n _ { j } j = k + 1 - m } }\end{subarray}} \prod_{l} \frac{1}{n_{l} !(l !)^{n_{l}}} C_{l}^{n_{l}}, \\
D_{m} & =\mathcal{O}(N),
\end{aligned}
$$

or

$$
\left\langle B H^{k}\right\rangle=\sum_{m=0}^{k}\left(\begin{array}{l}
k \\
m
\end{array}\right) D_{m+1}\left\langle H^{k-m}\right\rangle .
$$

On the basis of the formulas (11), (12) and (13) we now want to determine the order of magnitude of $\mathscr{D}_{i}$ and $\left\langle B e_{i}\right\rangle$, as given in (4) and (7) respectively.

We want to prove first two rather simple lemmas, which result in the statement that the series expansion in the second formula of (6) does not depend on $C_{1}$ and $D_{1}$. 
Lem ma I. The vector spaces spanned by the different sets $I, H, H^{2}, \ldots$ $H^{i-1}, i=1,2, \ldots, n$, do not depend on the value of $\langle H\rangle$, i.e. if a constant is added to the hamiltonian, this does not effect the different spaces. As a consequence $\mathscr{D}_{i}$ and $\left\langle B e_{i}\right\rangle$ are independent of $C_{1}$.

Proof: It is obvious that the sets $I, H+C, \ldots,(H+C)^{i-1}, i=1,2, \ldots$, $n$, are bases of vector spaces that are independent of $C, C$ being a real constant, because $(H+C)^{j}$ is simply a linear combination of all powers $H^{s}, s=0$, $1, \ldots j$. Therefore the orthogonalized powers of $H$, the basis vectors $e_{i}$, should be independent of a constant added to the hamiltonian and thus independent of $\langle H\rangle=C_{1} . \mathscr{D}_{i}$ may be expressed in terms of inner products of the $e_{i}$ and so these determinants cannot depend on $C_{1}$. For the $\left\langle B e_{i}\right\rangle$ it is immediately clear that they are independent of $C_{1}$.

Lemma II. The inner products $\left\langle B e_{i}\right\rangle$ are independent of $D_{1}$ for $i \geq 2$.

Proof: The coefficient of $D_{1}$ in the first row of the determinant in (7) are given respectively by $\langle I\rangle,\langle H\rangle, \ldots\left\langle H^{i-1}\right\rangle$, as follows from formula (13). These coefficients equal the corresponding elements of the second row, so one immediately sees that $\left\langle B e_{i}\right\rangle$ is independent of $D_{1}$. It is clear that one has to except the case $i=1$ for which $\left\langle B e_{i}\right\rangle=\langle B\rangle=D_{\mathbf{1}}$.

Combining both lemmas with formula (6) one may state that $\left\langle\left(B_{\text {diag }}-\langle B\rangle\right)^{2}\right\rangle$ is independent of $C_{1}$ and $D_{1}$. In addition to these lemmas we want to derive some further properties of $\left\langle H^{k}\right\rangle$ and $\left\langle B H^{k}\right\rangle$, properties based on the gencrating function for $\left\langle H^{k}\right\rangle$

$$
\begin{aligned}
& \left\langle H^{k}\right\rangle=\left\langle H^{k}(C)\right\rangle=\left[\left(\frac{\mathrm{d}}{\mathrm{d} t}\right)^{k} \exp \left(\sum_{i} \frac{C_{i}}{i !} t^{i}\right)\right]_{t=0} \\
& =\left[\left(\frac{\mathrm{d}}{\mathrm{d} t}\right)^{k} \exp G(t)\right]_{t=0}, \\
& G(t)=\sum_{i} \frac{C_{i}}{i !} t^{i}, \\
& \left\langle H^{k}(-C)\right\rangle=\left[\left(\frac{\mathrm{d}}{\mathrm{d} t}\right)^{k} \exp -G(t)\right]_{t=0} .
\end{aligned}
$$

In (16) $\left\langle H^{k}(-C)\right\rangle$ is introduced as a shorthand notation for the series (11) in which all $C_{l}$ are replaced by the corresponding $-C_{l}$. For the sake of clearness we write $\left\langle H^{k}(C)\right\rangle$ instead of $\left\langle H^{k}\right\rangle$ when it figures in a formula together with one of the $\left\langle H^{l}(-C)\right\rangle$.

On the basis of the formulas (14), (15) and (16) one easily proves a number of relations for the $\left\langle H^{k}\right\rangle$ and $\left\langle B H^{k}\right\rangle$, which will be given in the following lines. Mathematical details of the demonstration can be found in appendix 1 .

$$
\sum_{i=0}^{k}\left(\begin{array}{c}
k \\
i
\end{array}\right)\left\langle H^{i}(-C)\right\rangle\left\langle H^{k-i}(C)\right\rangle=0, \quad k \geq 1,
$$




$$
\begin{aligned}
& \sum_{i=0}^{k-1}\left(\begin{array}{c}
k-1 \\
i
\end{array}\right)\left\langle H^{i}(-C)\right\rangle\left\langle H^{k-i}(C)\right\rangle=C_{k}, \quad k \geq 1, \\
& \sum_{i=0}^{k-1}\left(\begin{array}{c}
k-1 \\
i
\end{array}\right)\left\langle H^{i}(-C)\right\rangle\left\langle B H^{k-1-i}(C)\right\rangle=D_{k}, \quad k \geq 1, \\
& f_{m, j}=\sum_{k=0}^{m-1} \sum_{i=0}^{j-1}\left(\begin{array}{c}
m-1 \\
k
\end{array}\right)\left(\begin{array}{c}
j-1 \\
l
\end{array}\right)\left\langle H^{k}(-C)\right\rangle\left\langle H^{l}(-C)\right\rangle\left\langle H^{m+j-2-k-l}(C)\right\rangle \\
& \quad=\mathscr{O}\left(N^{\min (m-1, j-1)}\right), \\
& f_{j, j} \sim(j-1) ! C_{2}^{j-1} .
\end{aligned}
$$

As the most important result of our analysis we now derive the order of magnitude of $\mathscr{D}_{i}$ and $\left\langle B e_{i}\right\rangle$, making use of formulas (I) to (V) and of wellknown properties of determinants

$$
\begin{aligned}
& \mathscr{D}_{i}=\left|d_{m, j}=\left\langle H^{m+j-2}\right\rangle ; 1 \leq m \leq i, 1 \leq j \leq i\right| \\
& =\left|e_{m, j}=\sum_{k=0}^{m-1}\left(\begin{array}{c}
m-1 \\
k
\end{array}\right)\left\langle H^{k}(-C)\right\rangle\left\langle H^{m+j}{ }^{2}{ }^{k}(C)\right\rangle\right| \\
& =\left|/ m, j=\sum_{k=0}^{m-1} \sum_{l=0}^{j-1}\left(\begin{array}{c}
m-1 \\
k
\end{array}\right)\left(\frac{i-1}{l}\right)\left\langle H^{k}(-C)\right\rangle\left\langle H^{l}(-C)\right\rangle\left\langle H^{m+j-2-k-l}(C)\right\rangle\right| \\
& \sim \prod_{j=1}^{i} f_{j, j} \sim \prod_{j=1}^{i-1} j ! C_{2}^{j}=C_{2}^{\frac{1}{i}(i-1)} \prod_{j=1}^{i-1} j !=\mathcal{O}\left(N^{\frac{1}{2}(i(i-1)}\right), \quad i \geq 1 \\
& \left\langle B e_{i}\right\rangle=\left|\begin{array}{l}
a_{1, j}=\left\langle B H^{j-1}\right\rangle_{i} 1 \leq j \leq i \\
a_{m, j}=\left\langle H^{m+j-3}\right\rangle ; 2 \leq m \leq i, 1 \leq j \leq i
\end{array}\right| \\
& =\left|\begin{array}{l}
b_{1, j}=\left\langle B H^{j-1}\right\rangle-\langle B\rangle\left\langle H^{j-1}\right\rangle ; 1 \leq j \leq i \\
b_{m, j}=\sum_{k=0}^{m-2}\left(\begin{array}{c}
m-2 \\
k
\end{array}\right)\left\langle H^{k}(-C)\right\rangle\left\langle H^{m+j-3-k}(C)\right\rangle ; 2 \leq m \leq i, 1 \leq j \leq i
\end{array}\right| \\
& =\left|\begin{array}{l}
c_{1, j}=\sum_{l=0}^{j-1}\left(\begin{array}{c}
j-1 \\
l
\end{array}\right)\left\langle H^{l}(-C)\right\rangle\left[\left\langle B H^{j-1-l}(C)\right\rangle-\langle B\rangle\left\langle H^{j-1-l}(\mathrm{C})\right\rangle\right] \\
c_{m, j}=\sum_{k=0}^{m-2} \sum_{l=0}^{j-1}\left(\begin{array}{c}
m-2 \\
k
\end{array}\right)\left({ }^{j-1} l_{l}\right)\left\langle H^{k}(-C)\right\rangle\left\langle H^{l}(-C)\right\rangle\left\langle H^{m+j-3-k-l}(C)\right\rangle
\end{array}\right| \\
& =\left|\begin{array}{cc:cccc}
0 & D_{2} & D_{3} & \ldots & \ldots & D_{i} \\
1 & 0 & 0 & \ldots & \ldots & 0 \\
0 & C_{2} & C_{3} & \cdots & \cdots & C_{i} \\
0 & C_{3} & & \multicolumn{2}{c}{c_{m, j}=t_{m-1, j}=\mathcal{O}\left(N^{\min (m-2, j-1)}\right)} \\
0 & C_{4} & & \\
\vdots & \vdots & & & \\
0 & C_{i-1} & & &
\end{array}\right| \\
& =\mathcal{O}\left(N^{1+1+2+3+\ldots i-2}\right)=\mathcal{O}\left(N^{\frac{1}{3}\left(i^{2}-3 i+4\right)}\right), \quad i \geq 2 .
\end{aligned}
$$


This formula turns out to be correct also for $i=1$

$$
\left\langle B e_{i}\right\rangle=\langle B\rangle=D_{1}=\mathcal{O}(N) .
$$

The formulas (18), (19) and (20) immediately give us the order of magnitude of the terms in the right-hand side of the second formula in (6)

$$
\frac{\left\langle B e_{i}\right\rangle^{2}}{\left\langle e_{i}^{2}\right\rangle}=\frac{\left\langle B e_{i}\right\rangle^{2}}{\mathscr{D}_{i-1} \mathscr{D}_{i}}=\mathscr{O}\left(N^{i^{2}-3 i+4-\frac{1}{2}(i-1)(i-2)-\frac{1}{2} i(i-1)}\right)=\mathscr{O}\left(N^{-i+3}\right) .
$$

As a consequence of (21) we are induced to replace the right-hand side of the second formula of (6), a sum of $n$ terms of increasing order in $1 / N$, by its first term and then find

$$
\begin{aligned}
& \left\langle\left(B_{\text {diag }}-\langle B\rangle\right)^{2}\right\rangle=\frac{[\langle B H\rangle-\langle B\rangle\langle H\rangle]^{2}}{\left\langle H^{2}\right\rangle-\frac{\langle H\rangle^{2}}{\langle}(1),} \\
& \lim _{N \rightarrow \infty} \frac{1}{N}\left\langle\left(B_{\text {diag }}-\langle B\rangle\right)^{2}\right\rangle=\lim _{N \rightarrow \infty} \frac{\chi_{0}-\chi_{\mathrm{s}}}{N},
\end{aligned}
$$

making use of formula (6) of Broer l.c. We know, however, that this result is incorrect for most models ${ }^{1-6}$ ), and that the left-hand side of (23) equals $\lim _{N \rightarrow \infty}\left(\chi_{0}-\chi_{\text {is }}\right) / N$ in all cases. The only possible conclusion is that the other terms in the sum $\sum_{i=2}^{n}\left\langle B e_{i}\right\rangle^{2} /\left\langle e_{i}^{2}\right\rangle$ together, give a contribution that is also of the order $\mathcal{O}(N)$ for these models. This is reasonable because of the fact that the number of levels $n=n(N)$ is a rapidly increasing function of $N$, in general of the form $a^{N}, a$ being a natural number. In addition to this one cannot be sure of the orders of magnitude given in (18) and (19) for $i$ of the order of $n$ because of the very large number of terms contributing to $\mathscr{D}_{i}$ and $\left\langle B e_{i}\right\rangle$. On the other hand we known that $\mathscr{D}_{n}=0$ for the case of one or more degeneracies and also $e_{n}=0$ in this case, and (18) and (19) give the wrong order of magnitude also here.

Those exceptional models for which (23) is correct may be called ergodic; they give the thermodynamical result. For these systems one may replace the series in (6) by the leading part of its first term.

We close this section by giving two examples of typical nonergodic systems for which it is easy to demonstrate that (22) and (23) are incorrect.

Example I. Systems composed of a number of uncoupled subsystems. We suppose that the different subsystems all have the same periodicity and consequently contain a number of particles that is of the order of $N$, the total number. For all the individual subsystems we may construct a basis as given in (2). If we call these bases $e_{i}^{(k)}, k=1,2, \ldots s$ numbering the different subsystems, a basis for the composed system is given by the set of all possible products $e_{i_{1}}^{(1)} e_{i_{2}}^{(2)} \ldots e_{i_{s}}^{(s)}$.

Now we make the supposition that all subsystems individually are ergodic 
and then we may write

$$
\left\langle\left(B_{\mathrm{diag}}-\langle B\rangle\right)^{2}\right\rangle \sim \sum_{k=1}^{s} \frac{\left\langle B e_{2}^{(k)}\right\rangle^{2}}{\left\langle e_{2}^{(k) 2}\right\rangle},
$$

all terms in the right-hand side of (24) being of the order $N$. The $e_{2}^{(k)}$ correspond with the hamiltonians of the (uncoupled) subsystems $H^{(k)}$,

$$
H=\sum_{k} H^{(k)}, \quad e_{2}^{(k)}=\left\langle H^{(k)}\right\rangle-H^{(k)} .
$$

In most cases $B$ is a sum of terms for the individual subsystems

$$
B=\sum_{k} B^{(k)},\left\langle B e_{2}^{(k)}\right\rangle=\left\langle B^{(k)} e_{2}^{(k)}\right\rangle .
$$

In general one has

$$
\frac{\left\langle B e_{2}\right\rangle^{2}}{\left\langle e_{2}^{2}\right\rangle}<\sum_{k} \frac{\left\langle B e_{2}^{(k)}\right\rangle^{2}}{\left.\left\langle e_{2}^{(k)}\right)^{2}\right\rangle}, \quad e_{2}=\sum_{k} e_{2}^{(k)},
$$

because of the invariance for an orthogonal transformation of the righthand side of the inequality; $e_{2}$ is just one single linear combination of the $e_{2}^{(k)}$. From (24) and (25) it immediately follows that these composed systems are generally nonergodic. A necessary and sufficient condition for ergodicity in this case can easily be formulated if we introduce the symbols

$$
\left\langle B e_{2}^{(k)}\right\rangle=x_{k}, \quad\left\langle e_{2}^{(k) 2}\right\rangle=a_{k}>0
$$

Now the condition is

$$
\left(\sum_{k} x_{k}\right)^{2} / \sum_{k} a_{k}=\sum_{k} x_{k}^{2} / a_{k} \quad \text { or } \quad x_{k} / a_{k}=c,
$$

$c$ being independent of $k$.

Example II. The $X-Y$ model. In examples like the $X-Y$ model, or the ferromagnet and the antiferromagnet in the spinwave approximation, we are able to indicate a set of $N$ diagonal operators corresponding with different modes $k=1,2 \ldots, N$.

In the $X$-Ymodel all modes give a contribution to $\left\langle\left(B_{\text {diag }}-B\right)^{2}\right\rangle$ of the order 1 , for $B=M_{z}$, the $z$ component of the total magnetization. We shall discuss this model in more detail, on the basis of the analysis made by Niemeijer ${ }^{8}$ ) and Mazur $^{1}$ ).

The $N$ diagonal operators in this example correspond with the $N$ different fermion modes, all reflecting the full translational symmetry. All modes together give a total contribution of order $\mathcal{O}(N)$ as follows from Mazur's analysis in section 4 of his paper

$$
\begin{gathered}
\left\langle\left(M_{z \text { diag }}-\left\langle M_{z}\right\rangle\right)^{2}\right\rangle=\sum_{k}\left\langle M_{z} e_{2}^{(k)}\right\rangle 2\left\langle\left\langle e_{2}^{(k) 2}\right\rangle=\mathcal{O}(N),\right. \\
e_{2}^{(k)}=n_{k}-\left\langle n_{k}\right\rangle, \quad k=1 \ldots N .
\end{gathered}
$$


One would expect that all individual modes generate a set analogous to the one of formula (2), but taking into account that the operators $n_{k}$ are idempotent i.e. $n_{k}^{2}=n_{k}$, one sees that the higher powers do not give independent diagonal operators. The crossterms $\left(n_{k_{1}}-\left\langle n_{k_{1}}\right\rangle\right)\left(n_{k_{2}}-\left\langle n_{k_{2}}\right\rangle\right), k_{1} \neq k_{2}$; $\left(n_{k_{1}}-\left\langle n_{k_{2}}\right\rangle\right)\left(n_{k_{2}}-\left\langle n_{k_{2}}\right\rangle\right)\left(n_{k_{3}}-\left\langle n_{k_{3}}\right\rangle\right), k_{1} k_{2} k_{3} \neq$, etc. do not give a contribution, $M_{z \text { diag }}$ being a linear combination of the $n_{k}$, which are orthogonal to all crossproducts. One immediately sees that the operators $I, e_{2}^{(k)} k=1,2$, $\ldots N$, combined with all crossproducts constitute a basis, their total number being $\sum_{l=0}^{N}\left(\begin{array}{l}N \\ l\end{array}\right)=2^{N}=n$.

If we had taken a series of orthogonalized powers of $H$ as our basic set $e_{i}$, and if we had used formula (21) and restricted ourselves to $e_{2}$, we would have found the wrong value for $\lim _{N \rightarrow \infty}\left\langle\left(M_{z \text { diag }}-\left\langle M_{z}\right\rangle\right)^{2}\right\rangle \mid N$ as follows from Mazur's analysis, or in other words $\chi_{\text {is }} \neq \chi_{\mathrm{s}}$. Here an inequality of the type (25) plays a role. In appendix 2 we give a necessary and sufficient condition for the exact equivalence of $\chi_{1 \mathrm{~s}}$ and $\chi_{\mathrm{s}}$ for models like the $X-Y$ model, for which the total hamiltonian may be written as a sum of mutually independent terms, which are functions of a parameter $h$.

4. Nonergodicity and level crossing. In most practical cxamples for which the diagonal part of an operator $B$ has to be determined, the hamiltonian of the system contains a term corresponding with the coupling of $B$ with an external field $h: H=A+h B$. The traditional example, with a finite number of states $n$, is the system of magnetic spins or dipoles with interaction. In a paper ${ }^{5}$ ) devoted to the discussion of the difference of $\chi_{\mathrm{s}}$ and $\chi_{\text {is }}$ the author introduced the noncrossing hypothesis in order to prove the ergodicity, or the asymptotic equivalence of $\chi_{\text {is }}$ and $\chi_{\mathrm{s}}$. Now we have found a more refined criterion, being the convergence of an infinite series, but we still believe that the noncrossing hypothesis has an intimate connection with the convergence or divergence of this series.

In a recent paper ${ }^{7}$ ) the author showed that crossings gave the possibility to transform the basis $e_{i}=(A+h B)^{i-1}, i=1, \ldots, n$, into a basis that also consists of polynomials in $h$, but some being of a degree that is lower than the one of the corresponding $e_{i}$. Composite systems having uncoupled parts are typical examples for which level crossings can be connected with the existence of nontrivial constants of motion, corresponding with the hamiltonians of these parts. The nonergodicity of these systems was discussed at length in the preceding section. One should remark that for large systems only a large number of crossings may result in such a reduction of the basis that there appear additional constants of motion of the type discussed in the preceding section.

For the hamiltonian $H=A+h B$ the expectation value of $B$ for an eigenstate corresponds with the slope of the energy eigenvalue as a function of $h$. In the case of ergodic systems this slope is a (quasi) continuous function 
of the energy, in other words the fluctuations of higher order in the energy do not contribute to the fluctuations in the slope, as is reflected in (21). One also understands that a large fluctuation of the slope results in a large number of crossings. There are systems, however, that have a large number of level crossings, e.g. an odd number of spins $\frac{1}{2}$ with interaction for which all levels are Kramers-degenerate for $h=0$, but the levels may still have a quasi-continuous slope, because the Kramers degeneracy is expected to be the only possible cause of crossing. As a rough estimate one may expect nonergodic behaviour only for a number of crossings of the order $n^{2}$.

Valkering ${ }^{9}$ ) recently developed a general algebraic criterion in terms of the properties of $A$ and $B$, for the existence of level crossings. His work is based on the analysis in ref. 7. This criterion may be applied to systems of such a number of levels that actual numerical calculation of the energy eigenvalues for a range of $h$ values does not seem possible. Especially for larger systems knowledge of the maximum number of level crossings is very interesting from the point of view of ergodic theory.

5. Uniform convergence and ergodicity. In formula (6) we have found a series expansion for the fluctuation of the diagonal part of the operator $B$

$$
\sum_{i=2}^{n(N)} \frac{1}{N} \frac{\left\langle B e_{i}\right\rangle^{2}}{\left\langle e_{i}^{2}\right\rangle}=\frac{1}{N}\left\langle\left\langle B_{\text {diag }}-\langle B\rangle\right)^{2}\right\rangle=F\left(\frac{1}{N}\right),
$$

in which every term can be written as an asymptotic expression in $1 / N$ after substitution of (18) and (19).

For a given set of systems, e.g. linear chains of $N$ spins with short-range interactions, $N=1,2,3 \ldots$, the limit for $N \rightarrow \infty$ of every term in the series may exist. Then a series expansion for every individual term may be found, which gives the correct value for every discrete $N$ with $n(N) \geq i$

$$
\begin{array}{rlrl}
\frac{1}{N} \frac{\left\langle B e_{i}\right\rangle^{2}}{\left\langle e_{i}^{2}\right\rangle} & =\frac{1}{N^{i-2}}\left(a_{i, 0}+\frac{a_{i, 1}}{N}+\frac{a_{i, 2}}{N^{2}}+\ldots\right), & & n(N) \geq i \geq 2, \\
& =0 \quad, \quad n(N)<i .
\end{array}
$$

This series expansion defines an analytic function in the open domain $|z|<1$

$$
\begin{aligned}
& f_{i}(z)=z^{i-2}\left(a_{i, 0}+a_{i, 1} z+a_{i, 2} z^{2}+\ldots\right), \\
& f_{i}(0)=\delta_{i 2} a_{2,0} .
\end{aligned}
$$

It may happen that there is degeneracy for a discrete value of $h$, i.e. a level crossing for $h=h_{0}$. Then there will be one or more $e_{i}\left(h_{0}\right)=0$. The values of $\left\langle B e_{i}\right\rangle^{2} /\left\langle e_{i}^{2}\right\rangle\left(h=h_{0}\right)$ for these $e_{i}$ may be defined by the proper limit $h \rightarrow h_{0}$. Now the property of ergodicity may be derived from the possible uniform 
convergence of the series $\sum_{i=2}^{\infty} f_{i}(z)$ on a discrete set

$$
z=\frac{1}{N}=\frac{1}{N_{0}}, \frac{1}{N_{0}+1}, \frac{1}{N_{0}+2}, \ldots
$$

including $1 / N=0(N=\infty)$.

For the case that there is uniform convergence and that the terms are analytic we can state that:

For every positive $\varepsilon$ we can find a positive integer $n_{0}$, independent of $N$, so that for $n>n_{0}$

$$
\left|F\left(\frac{1}{N}\right)-\sum_{i=2}^{n} \frac{1}{N} \frac{\left\langle B e_{i}\right\rangle^{2}}{\left\langle e_{i}^{\mathbf{2}}\right\rangle}\right|<\varepsilon .
$$

Taking the limit $N \rightarrow \infty$ in (32) or substituting $z=1 / N=0$ we find

$$
\left|F(0)-f_{2}(0)\right|<\varepsilon \quad \text { or } \quad k T\left|\lim _{N \rightarrow \infty} \frac{\chi_{0}-\chi_{\text {is }}}{N}-\lim _{N \rightarrow \infty} \frac{\chi_{0}-\chi_{\mathrm{s}}}{N}\right|<\varepsilon,
$$

which implies

$$
\lim _{N \rightarrow \infty} \frac{\chi \mathbf{s}-\chi_{\text {is }}}{N}=0
$$

The uniform convergence results in conditions for the quantities $C_{i}, D_{\hat{j}}$ introduced in section 3 . It can be shown with formula (19) that all $\left\langle B e_{i}\right\rangle$ are linear conbinations of the determinantal forms $C_{i} D_{j}-C_{j} D_{i}$, with coefficients that only depend on the $C_{k}$. All specific information about a particular system is contained in the value of the $C_{i}$ and $D_{j}$.

\section{APPENDIX 1}

Sum rules for $\left\langle H^{i}\right\rangle$ and $\left\langle B H^{i}\right\rangle$. The derivation of the formulas (17): I to $V$ of section 3 is based on the properties of the generating functions

$$
\exp G(t) \text { and } \exp -G(t)
$$

introduced in (14), (15) and (16).

$$
\begin{aligned}
\sum_{i=0}^{k}\left(\begin{array}{l}
k \\
i
\end{array}\right) & \left\langle H^{i}(-C)\right\rangle\left\langle H^{k-i}(C)\right\rangle \\
& =\sum_{i=0}^{k}\left(\begin{array}{c}
k \\
i
\end{array}\right)\left[\left(\frac{\mathrm{d}}{\mathrm{d} t_{1}}\right)^{i} \exp \left(-G\left(t_{1}\right)\right)\right]_{t_{1}=0}\left[\left(\frac{\mathrm{d}}{\mathrm{d} t_{2}}\right)^{k-i} \exp G\left(t_{2}\right)\right]_{t_{2}=0} \\
& =\sum_{i=0}^{k}\left(\begin{array}{c}
k \\
i
\end{array}\right)\left[\left(\frac{\mathrm{d}}{\mathrm{d} t}\right)^{i} \exp (-G(t))\left(\frac{\mathrm{d}}{\mathrm{d} t}\right)^{k-i} \exp G(t)\right]_{t=0} \\
& =\left.\left(\frac{\mathrm{d}}{\mathrm{d} t}\right)^{k} \exp (-G(t)) \exp G(t)\right|_{t=0}=0, \quad k \geq 1 .
\end{aligned}
$$


(II)

$$
\begin{aligned}
& \sum_{i=0}^{k-1}\left({ }_{i=1}^{k-1}\right)\left\langle H^{i}(-C)\right\rangle\left\langle H^{k-i}(C)\right\rangle \\
& =\sum_{i=0}^{k-1}\left({ }^{k-1}{ }_{i}\right)\left[\left(\frac{\mathrm{d}}{\mathrm{d} t_{1}}\right)^{i} \exp \left(-G\left(t_{1}\right)\right)\right]_{t_{1}=0}\left[\left(\frac{\mathrm{d}}{d t_{2}}\right)^{k-i} \exp G\left(t_{2}\right)\right]_{t_{2}=0} \\
& =\left\{\left(\frac{d}{\mathrm{~d} t}\right)^{k-1}\left[\exp (-G(t)) \frac{\mathrm{d}}{\mathrm{d} t} \exp G(t)\right]\right\}_{t=0} \\
& =\left.\left(\frac{\mathrm{d}}{\mathrm{d} t}\right)^{k} G(t)\right|_{t=0}=C_{k}, \quad k \geq 1 .
\end{aligned}
$$

(III) $\left\langle B H^{i}\right\rangle=i ! \sum_{m=1}^{i+1} \frac{D_{m}}{(m-1) !} \frac{\left\langle H^{i+1-m}\right\rangle}{(i+1-m) !}=\sum_{m=1}^{i+1}\left(\begin{array}{c}i \\ m-1\end{array}\right) D_{m}\left\langle H^{i+1-m}\right\rangle$, $k-1$

$\sum_{i=0}\left(\begin{array}{c}k-1 \\ i\end{array}\right)\left\langle H^{i}(-C)\right\rangle\left\langle B H^{k-1-i}(C)\right\rangle$

$$
\begin{aligned}
& =\sum_{i=0}^{k-1} \sum_{m=1}^{k-i}\left(\begin{array}{c}
k-1 \\
i
\end{array}\right)\left(\begin{array}{c}
k-1-i \\
m-1
\end{array}\right) D_{m}\left\langle H^{i}(-C)\right\rangle\left\langle H^{k-i-m}(C)\right\rangle, \\
& \sum_{i=0}^{k-1} \sum_{m=1}^{k-i} \ldots=\sum_{m=1}^{k} \sum_{i=0}^{k-m} \ldots, \quad\left(\begin{array}{c}
k-1 \\
i
\end{array}\right)\left(\begin{array}{c}
k-1-i \\
m-1
\end{array}\right)=\left(\begin{array}{c}
k-m \\
i
\end{array}\right)\left(\begin{array}{c}
k-1 \\
m-1
\end{array}\right), \\
& \sum_{i=0}^{k-1}\left(\begin{array}{c}
k-1 \\
i
\end{array}\right)\left\langle H^{i}(-C)\right\rangle\left\langle B H^{k-1-i}(C)\right\rangle \\
& =\sum_{m=1}^{k} \sum_{i=0}^{k-m}\left(\begin{array}{c}
k-m \\
i
\end{array}\right)\left(\begin{array}{c}
k-1 \\
m-1
\end{array}\right) D_{m}\left\langle H^{i}(-C)\right\rangle\left\langle H^{k-i-m}(C)\right\rangle \\
& =\sum_{m=1}^{k} \delta_{m k}\left(\begin{array}{c}
k-1 \\
m-1
\end{array}\right) D_{m}=D_{k}, \quad k \geq 1 .
\end{aligned}
$$

(IV) $\quad f_{m, j}=\left(\frac{\partial}{\partial t_{1}}+\frac{\partial}{\partial t_{3}}\right)^{m-1}\left(\frac{\partial}{\partial t_{2}}+\frac{\partial}{\partial t_{3}}\right)^{j-1}$

$$
\begin{aligned}
& \times \exp \left[-G\left(t_{1}\right)-G\left(t_{2}\right)+G\left(t_{3}\right)\right]_{t_{1}-t_{2}=t_{3}-0} \text {. } \\
& t_{1}=x, t_{2}=y, t_{3}=x+y+z \text {; } \\
& f_{m, j}=\left(\frac{\partial}{\partial x}\right)^{m-1}\left(\frac{\partial}{\partial y}\right)^{j-1} \exp [-G(x)-G(y)+G(x+y+z)]_{x=y=z=0} \\
& =\left(\frac{\partial}{\partial x}\right)^{m-1}\left(\frac{\partial}{\partial y}\right)^{j-1} \exp \left[-G(y)+G^{\prime}(x) y+G^{\prime \prime}(x) \frac{y^{2}}{2}+\ldots\right]_{x=y=0} \\
& =\left(\frac{\partial}{\partial x}\right)^{m-1}\left(\frac{\partial}{\partial y}\right)^{j-1} \exp \left[-G(0)+\sum_{k=1}^{\infty} \frac{G^{(k)}(x)-G^{(k)}(0)}{k !} y^{k}\right]_{x=y=0} \\
& -\mathcal{O}\left(N^{\min (m-1 . j-1)}\right) \text {. }
\end{aligned}
$$


(V)

$$
\begin{aligned}
f_{j, j} \sim & \left(\frac{\partial}{\partial x}\right)^{j-1}\left(\frac{\partial}{\partial y}\right)^{j-1} \exp \left[-C_{1} x-\frac{C_{2}}{2} x^{2}-C_{1} y-\frac{C_{2}}{2} y^{2}\right. \\
& \left.+C_{1}(x+y)+\frac{C_{2}}{2}(x+y)^{2}\right]\left.\right|_{x=y=0} \\
& =\left(\frac{\partial}{\partial x}\right)^{j-1}\left(\frac{\partial}{\partial y}\right)^{j-1} \exp C_{2} x y=(j-1) ! C_{2}^{j-1} .
\end{aligned}
$$

\section{APPENDIX 2}

$M_{z \text { diag }}$ for the $X$-Ymodel. In the $X$-Ymodel the hamiltonian in terms of fermion variables, apart from a constant, takes the form 1,8 )

$$
H=\sum_{k=1}^{N} \Lambda_{k} n_{k}
$$

$k$ corresponding with the different modes. Again two basis vectors of the space of diagonal operators are given by

$$
e_{1}=1, \quad e_{2}=H-\langle H\rangle=\sum_{k=1}^{N} A_{k}\left(n_{k}-\left\langle n_{k}\right\rangle\right),
$$

and we are interested in the contribution of $e_{2}$ to

$$
M_{z \mathrm{diag}}-\left\langle M_{z}\right\rangle=\sum_{k} M_{k}\left(n_{k}-\left\langle n_{k}\right\rangle\right), \quad M_{k}=-\partial \Lambda_{k} / \partial b, \quad h=-b
$$

$b$ corresponds to the external magnetic field $\left.{ }^{1,8}\right)$. From (A2.2) and (A2.3) it follows that

$$
\begin{aligned}
& \left\langle\left(M_{z \text { diag }}-\left\langle M_{z}\right\rangle\right)^{2}\right\rangle=\sum_{k} M_{k}^{2}\left(\left\langle n_{k}\right\rangle-\left\langle n_{k}\right\rangle^{2}\right), \quad\left\langle n_{k}^{2}\right\rangle=\left\langle n_{k}\right\rangle, \\
& \frac{\left\langle M e_{2}\right\rangle^{2}}{\left\langle e_{2}^{2}\right\rangle}=\frac{\left[\sum_{k} M_{k} A_{k}\left(\left\langle n_{k}\right\rangle-\left\langle n_{k}\right\rangle^{2}\right)\right]^{2}}{\sum_{k} \Lambda_{k}^{2}\left(\left\langle n_{k}\right\rangle-\left\langle n_{k}\right\rangle^{2}\right)} .
\end{aligned}
$$

For the exact equivalence of $\chi_{\text {is }}$ and $\chi_{\mathrm{s}}$ we should have

$$
\sum_{k} M_{k}^{2}\left(\left\langle n_{k}\right\rangle-\left\langle n_{k}\right\rangle^{2}\right)=\frac{\left[\sum_{k} M_{k} \Lambda_{k}\left(\left\langle n_{k}\right\rangle-\left\langle n_{k}\right\rangle^{2}\right)\right]^{2}}{\sum_{k} \Lambda_{k}^{2}\left(\left\langle n_{k}\right\rangle-\left\langle n_{k}\right\rangle^{2}\right)},
$$

which identity implies $M_{k}=C \Lambda_{k}, C$ being a constant independent of $k$. This constant, however, should be a function of $b$, the magnetic field and taking into account the second formula in (A2.3), we find the following 
expression for the logarithmic derivative of the $\Lambda_{k}$

$$
\frac{\partial \Lambda_{k}}{\partial b} / \Lambda_{k}=-C(b)
$$

which gives after integration

$$
\Lambda_{k}=c_{k} F(b), \quad F(b)=\exp -\int C(b) \mathrm{d} b .
$$

The actual integration of the equations of motion for the $X-Y$ model does not lead to elementary excitations of the type (A2.8), but we already knew that this model is not ergodic. For such a model, however, with a hamiltonian that can be written in the form (A2.1) the greater part of the elementary excitations should asymptotically, be given by (A2.8). As follows from the analysis in section I.4.A of ref. $2 F(b)$ should have the form

$$
F(b)=\left(a+c b^{2}\right)^{\frac{1}{2}},
$$

for this case.

\section{REFERENCES}

1) Mazur, P., Physica 43 (1969) 533.

2) Caspers, W. J., Theory of spin relaxation, Tnterscience Publ. (New York, 1964).

3) Broer, L. J. F., Physica 17 (1951) 531.

4) Yamamoto, T., Phys. Rev. 119 (1960) 701.

5) Caspers, W. J., Physica 25 (1959) 645.

6) Klein, M. J., Phys. Rev. 86 (1952) 807.

7) Caspers, W. J., Physica 40 (1968) 125.

8) Niemeijer, Th., Physica 36 (1967) 377.

9) Valkering, T. P., Physica 53 (1971) 117.

10) Kubo, R., J. Phys. Soc. Japan 17 (1962) 1100. 